\title{
Improved distributed-index planar microlens and its application to 2-D lightwave components
}

\author{
Masahiro Oikawa, Kenichi Iga, Shigeyoshi Misawa, and Yasuo Kokubun
}

\begin{abstract}
A recent improvement of a 2-D array of distributed-index (DI) planar microlenses is presented to demonstrate a 2-D integrated optical component using a planar microlens array. These components are made by stacking planar microlens arrays and other planar optical devices. The improved planar microlens has $f=$ $2.0 \mathrm{~mm}$, a N.A. $=0.23$, and its spherical aberration is very small. A fiber coupler is demonstrated. From a preliminary experiment, it is shown that the coupling loss of each channel is $<0.5 \mathrm{~dB}$ for coupling $50-\mu \mathrm{m}$ core DI fibers.
\end{abstract}

\section{Introduction}

Integration and mass production of lightwave components are important in large capacity fiber transmission systems. However, since conventional microoptics 1,2 is constructed with discrete lenses such as distributed-index (DI) rod lenses (or GRIN rods) and spherical microlenses, a number of aligning processes are required which prevent easy preparation of integrated components. We proposed a stacked planar optics $^{3,4}$ by using a 2-D planar microlens array, where we construct 2-D components by stacking planar optical devices. The only problem to be solved is how to increase the N.A. of the microlens enough to cover the N.A. of the optical fibers. In this study, we have greatly improved the performance of the planar microlens and present some uses for the lightwave microoptic components.

\section{Improved Planar Microlens}

We have developed a DI planar microlens ${ }^{5-8}$ which is fabricated by field-assisted diffusion of metal ions into a planar glass substrate as shown in Fig. 1. However, the N.A. of the lens was not enough (N.A. 0.17) to accept light from the optical fibers. Recently we succeeded in making a $0.9-\mathrm{mm}$ diam planar microlens with N.A. $=0.23$ and focal length $f=2.0 \mathrm{~mm}$. The N.A. was increased as follows: (1) by a better choice of molten salt consisting of metal ions; (2) by the optimization of electromigration conditions such as temper-

The authors are with Tokyo Institute of Technology, 4259 Nagatsuta, Midori-ku, Yokohama 227, Japan.

Received 21 September 1982.

0003-6935/83/030441-02\$01.00/0.

(C) 1983 Optical Society of America. ature, time, and applied voltage; and (3) by the shortened annealing time. The substrate of the planar microlens was a planar $40-\times 40-\times 4-\mathrm{mm}^{3}$ glass where windows of $100-\mu \mathrm{m}$ diffusion mask are prepared as an $11 \times 17$ matrix with a pitch of $2 \mathrm{~mm}$. The improved planar microlens was fabricated by a few hours of electromigration. We measured the refractive-index profile of the planar microlens using a Mach-Zehnder interference microscope. ${ }^{9}$ The interference patterns from a longitudinal thin plate, $50 \mu \mathrm{m}$ thick including the center axis, indicate equi-index curves as shown in Fig. 1(b). The index profile is almost hemispherical with maximum index difference of $\Delta n=0.13$. The resulting N.A. was 0.23 which was large enough to accept the light from an ordinary multimode fiber whose N.A. is around 0.2 . We stacked two planar microlenses to obtain a larger N.A. The N.A. was 0.38 with $1.8-\mathrm{mm}$ focal length in a glass substrate with refractive index of $n_{2}=1.52$. This N.A. almost reaches that of a commercially available DI rod lens. ${ }^{10}$ We focused a $\mathrm{He}-\mathrm{Ne}$ laser beam $(\lambda$ $=0.63 \mu \mathrm{m}$ ) to a $3.8-\mu \mathrm{m}$ diam spot using a piece of stacked planar microlens as shown in Fig. 2. A circularly symmetric focused spot also indicated that asymmetry of the lens is very small.

\section{Application to Lightwave Components}

By using the 2-D array of planar microlenses, a lot of lightwave components can be made, as we proposed as a stacked planar optics. $3,4,11$

We made a $2 \times 2$ fiber coupler array by using improved planar microlenses as shown in Fig. 3 . The coupler array is composed of two lens elements: one collimates the light from an input fiber and the other focuses the collimated light into an output fiber. These two elements have the same structure and are made of two lens arrays to have enough N.A. The length of one element is $2 \mathrm{~mm}$, designed to locate a fiber near the 


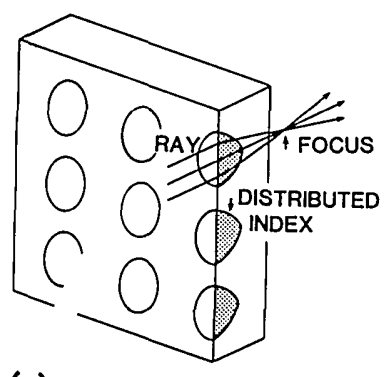

(a)

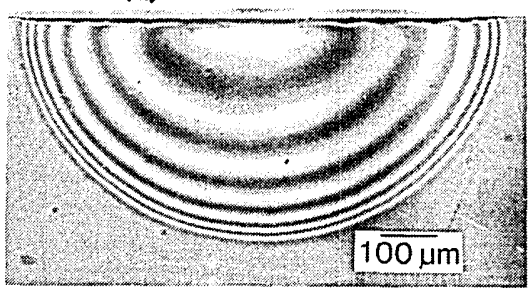

(b)

Fig. 1. (a) Configuration of a DI planar microlens. (b) Interference pattern which shows equi-index surfaces in the meridional plane.

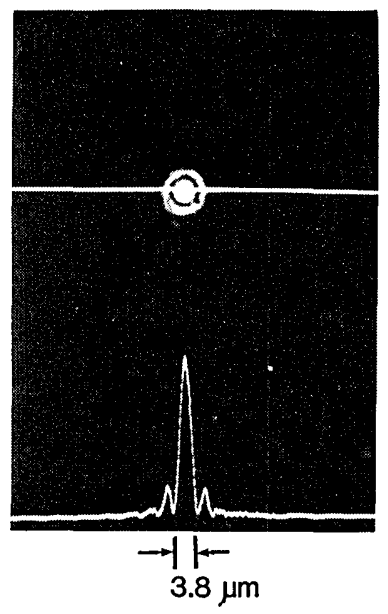

Fig. 2. Focused spot by the coupled planar microlens. A He-Ne laser beam $(\lambda=0.63 \mu \mathrm{m})$ was used as the light source.

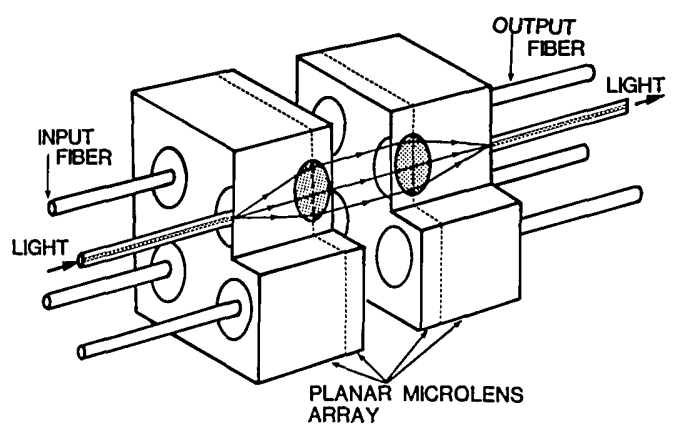

Fig. 3. Structure of the $2 \times 2$ fiber coupler array.
Table I. Coupling Loss of the Coupler Array

\begin{tabular}{cc}
\hline $\begin{array}{c}\text { Channel } \\
\text { no. }\end{array}$ & $\begin{array}{c}\text { Loss } \\
(\mathrm{dB})\end{array}$ \\
\hline 1 & 0.46 \\
2 & 0.51 \\
3 & 0.56 \\
4 & 0.51 \\
Average & 0.51 \\
\hline
\end{tabular}

surface. Multimode DI fibers (50- $\mu \mathrm{m}$ core) with N.A. of 0.23 were used. The input fiber was uniformly excited with a He-Ne laser $(\lambda=0.63 \mu \mathrm{m})$, and the output was monitored by Si solar cells. The average coupling loss was $0.51 \mathrm{~dB}$ with the best value of $0.46 \mathrm{~dB}$. The results are summarized in Table $\mathrm{I}$. This could be improved when the modes are in a steady state and the fibers are carefully located at an optimum position.

\section{Summary}

Improvement of a planar microlens and its application to lightwave components have been demonstrated. Low coupling loss of the $2 \times 2$ coupler array indicates a possibility of $2-\mathrm{D}$ array and mass production of lightwave components by using the configuration of stacked planar optics.

We would like to acknowledge Y. Suematsu, T. Tako, N. Ooura, and I. Shimizu for supporting this work. We also thank I. Kitano, T. Yamazaki, K. Nishizawa, and N. Yamamoto, Nippon Sheet Glass Co., Ltd., for discussion, and we are indebted to K. Inada, Fujikura Cable Works Co., Ltd. for supplying some of the optical fibers.

\section{References}

1. K. Kobayashi, R. Ishikawa, K. Minemura, and S. Sugimoto, Fiber Integ. Opt. 2, 1 (1979).

2. W. J. Tomlinson, Appl. Opt. 19, 1127 (1980).

3. K. Iga, M. Oikawa, S. Misawa, and J. Banno, in Digest of Topical Meeting on Integrated and Guided-Wave Optics (Optical Society of America, Washington, D.C., 1982), paper FB6.

4. K. Iga, M. Oikawa, S. Misawa, J. Banno, and Y. Kokubun, Appl. Opt. 21, 3456 (1982).

5. M. Oikawa, K. Iga, and T. Sanada, Jpn. J. Appl. Phys. 20, L51 (1980).

6. M. Oikawa, K. Iga, T. Sanada, N. Yamamoto, and K. Nishizawa, Jpn. J. Appl. Phys. 20, L296 (1981).

7. M. Oikawa, K. Iga, and T. Sanada, Electron. Lett. 17, 452 (1981).

8. M. Oikawa and K. Iga, Appl. Opt. 21, 1052 (1982).

9. Y. Kokubun and K. Iga, Appl. Opt. 21, 1030 (1982).

10. N. Akazawa, M. Toyama, and K. Matsushita, in Digest of Topical Meeting on Gradient-Index Optical Imaging Systems (Optical Society of America, Washington, D.C., 1981), paper TuD4. Data sheet of Selfoc lens, Nippon Sheet Glass Co., Ltd.

11. M. Oikawa, K. Iga, and S. Misawa, Electron. Lett. 18, 316 (1982). 